\title{
The Existence of Chern-Simons Vortices
}

\author{
Ronggang Wang \\ Department of Mathematics, Harvard University, Cambridge, MA 02138, USA
}

Received September 26, 1990; in revised form November 1, 1990

\begin{abstract}
A new type of vortices called Chern-Simons vortices is considered and the existence is established.
\end{abstract}

\section{Introduction}

Various vortex theories have been discussed in recent years, among them the classical, electrically charged and other ones. The discussions on vortices have proved important in quantum physics, solid state physics as well as in mathematics. A detailed account on classical (kinetic) vortex theory can be found in [14] by Jaffe and Taubes. When the Chern-Simons term is added to the action, this is $(2+1)$-dimensional massive electrodynamics, the vortex fields still exist and carry a fractional electric charge proportional to the coefficient of the Chern-Simons term. Such particles are called anyons, see Fröhlich and Marchetti [6].

The paper concerns the existence of Chern-Simons vortices recently found by physicists Jackiw and Weinberg, also Hong, Kim, and Pac who consider the problem of charged vortices with the gauge field governed solely by the ChernSimons term. This truncation is physically sensible at large distances and low energies, where the Chern-Simons term dominates the higher-derivative Maxwell term. With the symmetry breaking realization, it is interesting that with a special choice of the Higgs potential the vortex solutions satisfy a Bogomolny type "selfdual" equation. We call the solutions Chern-Simons vortices, see [10] also [18].

Chern-Simons vortex solutions can be compared to the classical vortex solutions and anyons. Chern-Simons vortices satisfy a similar "self-dual" equation as classical vortices but the equation contains a more complicated nonlinear term. The existence of axial solutions to the equation have been given in [10] and with a special choice of metric on the plane the equation is completely solvable [16]. In this paper, along the course of [14], a complex analytical and variational method is used to establish the general existence of solutions to the Chern-Simons vortex equation. Denote by $C$ or $R^{2}$ the standard 2-plane. 
Theorem. For any $\left(z_{1}, \ldots, z_{n}\right) \in C^{n}$, a charge $n$ smooth solution to the Chern-Simons vortex equation exists with zeros exactly at $z_{1}, \ldots, z_{n}$.

The main part of the paper is the analytical and variational problems raised from the Chern-Simons equation. The analysis in the problem in some sense is rather interesting; indeed, the Chern-Simons vortex equation as well as the classical vortex equation which is a complicated system of differential equations can be reduced by gauge transformation to a second order, variational, elliptic equation. In the Chern-Simons case, the variational problem can be compared to the prominent problem of Berger of prescribed scalar curvature on the 2-sphere; compared to the classical case the variational problem is more complicated and actually quite different but both can be regarded as examples of weak convergence method in the theory of partial differential equations. And due to the complexity of the variational problem, whether the solution to the Chern-Simons vortex equation is uniquely determined by its zeros remains unknown.

The paper is organized as follows. Section 2 is a brief review on the ChernSimons vortex equation and some fundamental properties of Chern-Simons vortices. In Sect. 3 the existence problem is converted into a variational problem and in Sect. 4 the variational problem is discussed.

The author would like to thank Professor Taubes for introducing him to the problem.

\section{The Chern-Simons Vortex Equation}

In this section we briefly recall the Chern-Simons vortex solutions and collect some fundamental properties of the solutions such as the counting of the zeros of a Chern-Simons vortex and the maximal principle of the Higgs field. The first half of the section is essentially based on [10] and the second part is a parallelism to the classical case. The interested reader may refer to $[10,14,15]$.

The Lagrangian density of the model (after a scaling of constants) is given by

$$
\mathscr{L}=|(d-i A) \phi|^{2}+\frac{1}{2} \varepsilon^{\alpha \beta \gamma} F_{\alpha \beta} A_{\gamma}-\frac{1}{4}|\phi|^{2}\left(1-|\phi|^{2}\right)^{2},
$$

where $\phi$ is a complex scalar field, $A$ a real gauge field, i.e.,

$$
A=A_{\mu} d x^{\mu} \text {, with } A_{\mu} \in R,
$$

and field strength $F=F_{\alpha \beta} d x^{\alpha} d x^{\beta}$ and the Chern-Simons term is

$$
\mathscr{L}_{\text {C.s. }}=\frac{1}{2} \varepsilon^{\alpha \beta \gamma} F_{\alpha \beta} A_{\gamma}
$$

with the Levi-Civita tensor $\varepsilon^{\alpha \beta \gamma}$ is fixed by $\varepsilon^{012}=1$.

Consider the motion defined by the Lagrangian (2.1), for time-independent configuration $(\phi, A)$ the critical condition gives

$$
A_{0}=-\frac{F_{12}}{|\phi|^{2}} \text {. }
$$

The action of the time-independent configuration $(\phi, A)$ with $A_{0}$ as (2.2) is given by the formula

$$
E(\phi, A)=\int_{x_{0}=0} d^{2} x(-\mathscr{L})=\int_{R^{2}}|(d-i A) \phi|^{2}+\frac{F_{12}^{2}}{|\phi|^{2}}+\frac{1}{4}|\phi|^{2}\left(1-|\phi|^{2}\right)^{2} .
$$


Assuming that the time-independent configuration satisfies the following boundary condition:

$$
\begin{gathered}
\lim _{R \rightarrow \infty} \sup _{|z| \leqq R}|1-| \phi||=0, \\
|z|^{1+\delta}|(d-i A) \phi| \leqq \mathrm{const}
\end{gathered}
$$

for some positive constant $\delta$, the energy functional $E(\phi, A)$ can be rewritten by a process of completing the square and integration by parts. As a matter of fact,

$$
\int \frac{F_{12}^{2}}{|\phi|^{2}}+\frac{1}{4}|\phi|^{2}\left(1-|\phi|^{2}\right)^{2}=\int\left|\frac{F_{12}}{\phi}-\frac{1}{2} \bar{\phi}\left(1-|\phi|^{2}\right)\right|^{2}+F_{12}\left(1-|\phi|^{2}\right)
$$

and

$$
\begin{aligned}
\int|(d-i A) \phi|^{2} & =\int\left|\left(D_{1}+i D_{2}\right) \phi\right|^{2}+i\left(D_{1} \phi D_{2}^{-} \phi-D_{2} \phi D_{1}^{-} \phi\right) \\
& =\int\left|\left(D_{1}+i D_{2}\right) \phi\right|^{2}+F_{12}|\phi|^{2}
\end{aligned}
$$

with the boundary term vanishing by the boundary condition. Thus with the boundary condition the energy functional can be rewritten as follows:

$$
E(\phi, A)=\int\left|\left(D_{1} \pm i D_{2}\right) \phi\right|^{2}+\left|\frac{F_{12}}{\phi} \mp \frac{1}{2} \bar{\phi}\left(1-|\phi|^{2}\right)\right|^{2} \pm \int F_{12} .
$$

With the boundary condition assumed as above, the vortex number of a configuration

$$
N=\frac{1}{2 \pi} \int F_{12}
$$

can be shown to be an integer as in the classical case; indeed, the number $N$ coincides with the degree defined by the map

$$
\left.\frac{\phi}{|\phi|}\right|_{|z|=R}: S^{1} \rightarrow S^{1}
$$

for $R$ sufficiently large.

As the classical case, finite energy configurations with the boundary condition fall into different path components labelled by the vortex number. On each path component, the energy functional $E$ has a lower bound:

$$
E \geqq 2 \pi|N|
$$

and the lower bound is achieved if and only if the configuration satisfies the following Chern-Simons vortex equation:

$$
\left\{\begin{array}{l}
\left(D_{1}+i D_{2}\right) \phi=0 \\
F_{12}=\frac{1}{2}|\phi|^{2}\left(1-|\phi|^{2}\right)
\end{array}\right.
$$

in the case $N>0$. The case $N<0$ is given by (2.4) after a sign change.

Chern-Simons vortex equation (2.4) can be regarded as a combination of a "complex" equation (2.4A) and a nonlinear equation (2.4B). To consider Eq. (2.4A), it is convenient to introduce the complex variables

$$
\begin{gathered}
z=x_{1}+i x_{2}, \quad A=\alpha d z+\bar{\alpha} d \bar{z}, \\
\partial_{z}=\frac{1}{2}\left(\partial_{1}-i \partial_{2}\right), \quad \partial_{\bar{z}}=\frac{1}{2}\left(\partial_{1}-i \partial_{2}\right),
\end{gathered}
$$


where

$$
\alpha=\frac{1}{2}\left(A_{1}-i A_{2}\right) .
$$

Under this convention, Eq. (2.4A) becomes a $\bar{\partial}$-equation

$$
\partial_{\bar{z}} \phi=i \bar{\alpha} \phi \text {. }
$$

The $\bar{\partial}$-Poincaré Lemma can be used to investigate the zeros of scalar field $\phi$.

Theorem 2.1. Let $(\phi, A)$ be a smooth Chern-Simons vortex with finite action, then

i) $\phi$ has finite number of zeros $z_{1}, \ldots, z_{m}$.

ii) Around each $z_{k}$, $\phi$ can be written as

$$
\phi=\left(z-z_{k}\right)^{n_{k}} h_{k}(z)
$$

with $h_{k}$ a smooth function, $h_{k}\left(z_{k}\right) \neq 0$.

iii) The vortex number $N$, is $\sum_{k=1}^{m} n_{k}$.

Proof. This is similar to the classical case, see III.5 of [14].

The other important property on Chern-Simons vortices is the fact that the Higgs field satisfies the maximum principle on the plane.

Theorem 2.2. Let $(\phi, A)$ be a smooth Chern-Simons vortex with the boundary condition, then $|\phi|<1$ on the whole plane.

Proof. It is convenient to consider the function

Taking the derivative,

$$
w=\frac{1}{2}\left(1-|\phi|^{2}\right) \text {. }
$$

$$
\begin{gathered}
d w=-\left(\phi, \nabla_{A} \phi\right), \\
\Delta w=-\left|\nabla_{A} \phi\right|^{2}+\left(\phi, \nabla_{A}^{*} \nabla_{A} \phi\right) \leqq\left(\phi, \nabla_{A}^{*} \nabla_{A} \phi\right) .
\end{gathered}
$$

Using the vortex equations and note that

on 1-forms on $R^{2}$,

$$
\nabla_{A}^{*}=D_{A}^{*}=-* D_{A} *
$$

$$
\nabla_{A}^{*} \nabla_{A} \phi=\frac{1}{2}|\phi|^{2}\left(1-|\phi|^{2}\right) \phi \text {. }
$$

The substitution yields

$$
\Delta w \leqq\left(\phi, \nabla_{A}^{*} \nabla_{A} \phi\right)=|\phi|^{4} w,
$$

and the maximum principle implies the fact that $|\phi|<1$.

The maximum principle will be seen more clearly as the vortex equation is reduced to a second order elliptic equation in Sect. 4.

\section{The Reduction of Chern-Simons Vortex Equation}

Chern-Simons vortex equation (2.4) is a system of differential equations with the complex scalar field $\phi$ and the gauge field $A$ as unknowns. In this section we reduce the equation to a variational problem of real functions. The reduction is divided as two steps: First the equation is reduced to a singular second order elliptic differential equation using a gauge transformation. The second step is to reduce the singular second order elliptic equation to a second order elliptic equation by choosing a reference solution. We see that the equation can be regarded as the 
Euler-Lagrange equation of a variational problem. The whole process is also parallel to the classical case.

First notice the fact that (2.5) can be rewritten as a $\partial$-equation

$$
\alpha=i \partial_{z} \ln \bar{\phi}
$$

on the area $\phi$ is nonzero. However, since $\alpha$ is smooth, it can be extended over the zeros of $\phi$ and we see that the gauge field $A$ is determined by the Higgs field $\phi$.

Writing the Higgs field as $\phi=e^{\frac{2}{2}(u+i \theta)}$, Theorems 2.1 and 2.2 say that $u$ is then a negative function with negative infinity as the value at the zeros of the Higgs field $\phi$, where $\theta$ is not defined. Furthermore, the gauge group acts on $\theta$ by adding to it a smooth function on $R^{2}$, or in another word, $\theta$ is defined by modulo a smooth function. Motivated by Theorem 2.1, denote

$$
\theta=2 \sum_{k=1}^{N} \arg \left(z-z_{k}\right) \text {. }
$$

The Chern-Simons vortex equation is equivalent to the following singular second order elliptic equation:

$$
\Delta u=e^{u}\left(e^{u}-1\right)+4 \pi \sum_{k=1}^{N} \delta\left(z-z_{k}\right)
$$

with the boundary condition $\lim _{|z| \rightarrow \infty} u=0$. Here, $\delta$ is the Dirac function.

Let $u_{0}$ be the following reference solution

$$
u_{0}=-\sum_{k=1}^{N} \ln \left(1+\mu\left|z-z_{k}\right|^{-2}\right) .
$$

With the real parameter $\mu$ varies $u_{0}$ is actually a family of reference solutions. For each $\mu>0, u_{0}$ is a negative function on the plane and $e^{u_{0}}$ vanishes with the same order as $|\phi|^{2}$. A calculation gives

$$
\Delta u_{0}=-4 \sum_{k=1}^{N} \frac{\mu}{\left(\mu+\left|z-z_{k}\right|^{2}\right)^{2}}+4 \pi \sum_{k=1}^{N} \delta\left(z-z_{k}\right) .
$$

By combining (3.2) and (3.4), in terms of $v=u-u_{0}$, Eq. (3.2) has the following equivalent form:

$$
\Delta v=e^{u_{0}+v}\left(e^{u_{0}+v}-1\right)+4 \sum_{k=1}^{N} \frac{\mu}{\left(\mu+\left|z-z_{k}\right|^{2}\right)^{2}} .
$$

Equation (3.5) is a second order elliptic partial differential equation with a nonlinearity term $e^{u_{0}+v}\left(e^{u_{0}+v}-1\right)$ and the boundary condition $\lim _{|=| \rightarrow \infty} v=0$. Denote

$$
g_{0}=4 \sum_{k=1}^{N} \frac{\mu}{\left(\mu+\left|z-z_{k}\right|^{2}\right)^{2}}
$$

with $\mu>0$ the real parameter, the second order equation (3.5) is then the EulerLagrange equation of the following functional:

$$
F(v)=\frac{1}{2} \int|\nabla v|^{2}+\left(e^{u_{0}+v}-1\right)^{2}+2 g_{0} v .
$$

This is our starting point of Sect. 4. 


\section{The Variational Problem and the Solution}

In this section we will concentrate on the variational problem (3.6). The solution of the variational problem (3.6) needs a set up of Banach spaces. It turns out that it is appropriate to consider the variational problem of the functional (3.6) on the space $H_{1}$, where $H_{1}$ is the completion of $C_{0}^{\infty}$-functions on the plane with the norm given by

$$
\|v\|^{2}=\int\left(|v|^{2}+|\nabla v|^{2}\right)
$$

Denote also $\|v\|_{p}$ the $L^{p}$-norm of a function $v$.

Lemma 4.1. The function $g_{0}$ is $L^{2}$-integrable with

$$
\left\|g_{0}\right\|_{2} \leqq \frac{C_{0}}{\sqrt{\mu}}
$$

for some positive constant $C_{0}$ independent of $\mu$ and $\left(e^{u_{0}}-1\right)$ is $L^{p}$-integrable for $p \geqq 2$. Proof.

$$
e^{u_{0}}=\prod_{k=1}^{N} \frac{1}{1+\mu\left|z-z_{k}\right|^{-2}} \leqq 1
$$

is a smooth function on the plane, $e^{u_{0}}-1$ approaches zero at infinity in an order of

$$
-\mu \sum_{k=1}^{N}\left|z-z_{k}\right|^{-2}
$$

which is equivalent to $-\frac{\mu N}{r^{2}}$. Thus $e^{u_{0}}-1$ is $L^{p}$-integrable $(p \geqq 2)$. The $L^{2}-$ integrability of $g_{0}$ is clear with

$$
\begin{aligned}
\int g_{0}^{2} & =\int\left(\sum_{k=1}^{N} \frac{\mu}{\left(\mu+\left|z-z_{k}\right|^{2}\right)^{2}}\right)^{2} \leqq N \sum_{k=1}^{N} \int \frac{\mu^{2}}{\left(\mu+\left|z-z_{k}\right|^{2}\right)^{4}} \\
& =N^{2} \int \frac{\mu^{2}}{\left(\mu+r^{2}\right)^{4}}=2 \pi N^{2} \int \frac{\mu^{2} r d r}{\left(\mu+r^{2}\right)^{4}}=\frac{2 \pi N^{2}}{\mu} \int \frac{r d r}{\left(1+r^{2}\right)^{4}} .
\end{aligned}
$$

Thus,

Return to the functional

$$
\left\|g_{0}\right\|_{2} \leqq \frac{C_{0}}{\sqrt{\mu}}
$$

$$
F(v)=\frac{1}{2} \int\left(|\nabla v|^{2}+\left(e^{u_{0}+v}-1\right)^{2}+2 g_{0} v\right) .
$$

$F(v)$ is a functional on $H_{1}$ with exponential nonlinearity and thus an estimate on the nonlinearity is necessary.

Lemma 4.2. On $R^{2}$, any function $v \in H_{1}$ satisfies the following:

$$
\|v\|_{p} \leqq\left\{\pi\left(\frac{p-2}{2}\right)\right\}^{\frac{p-2}{2 p}}\|v\|(p \geqq 2) .
$$

Proof. The proof can be found in cf. Sect. VI.3 of [12]. 
Lemma 4.3. On $R^{2}$, any $v \in H_{1}$ satisfies the following:

$$
\left\|\left(e^{v}-1\right)\right\|_{2}^{2} \leqq C_{1} \exp \left(C_{1}\|v\|^{2}\right)
$$

for some positive constant $C_{1}$.

Proof.

$$
\int\left(e^{v}-1\right)^{2}=\int v^{2}\left(\sum_{k=0}^{\infty} \frac{v^{k}}{(k+1) !}\right)^{2} \leqq \int v^{2}\left(\sum_{k=0}^{\infty} \frac{|v|^{k}}{k !}\right)^{2} \leqq \int v^{2} e^{2|v|} \leqq \sum_{k=0}^{N} \frac{2^{k}}{k !} \int|v|^{k+2} .
$$

Applying Lemma 4.2 and Stirlings Formula, there are positive constants $C_{2}$ and $C_{3}$ such that

$$
\left(C_{2} n\right)^{n} \leqq n ! \leqq\left(C_{3} n\right)^{n}
$$

sum up each term in (4.3) and get Lemma 4.3.

Restricted to a compact area on the plane, a slightly stronger result shows that the map $v \rightarrow\left(e^{v}-1\right)$ from $H_{1}$ to $L^{2}(K)$ is a compact map where $K$ is a compact set on the plane. We will not give the proof here; the interested reader may refer to Chap. 2, Sect. 15 of [1].

Thus the functional $F(v)$ is a continuous functional on $H_{1}$ by the fact that

$$
\begin{gathered}
\left|\int g_{0} v\right| \leqq\left(\int g_{0}^{2}\right)^{1 / 2}\left(\int v^{2}\right)^{1 / 2} \\
\int\left(e^{u_{0}+v}-1\right)^{2} \\
=\int\left(e^{u_{0}}\left(e^{v}-1\right)+\left(e^{u_{0}}-1\right)\right)^{2} \leqq 2 \int\left(e^{v}-1\right)^{2}+2 \int\left(e^{u_{0}}-1\right)^{2} \\
\leqq 2 C_{1} \exp \left(C_{1}\|v\|^{2}\right)+C_{4}
\end{gathered}
$$

for some constant $C_{4}>0$.

In our problem it is important to consider the weak (semi)-continuity of the functional $F(v)$.

Proposition 4.4. $F(v)$ is a weakly lower semi-continuous functional on $H_{1}$.

Proof. First note that the map $v \rightarrow\|v\|_{2}$ is a weakly lower semi-continuous functional on $H_{1}$. For any weakly convergent sequence $\left\{v_{n}\right\}$ to $v$ in $H_{1}$

$$
\int g_{0} v_{n} \rightarrow \int g_{0} v \text {. }
$$

Restricted on any compact area $K$ on the plane, $\lim \int_{K}\left(e^{v_{n}}-1\right)^{2}$ exists and equals to
$\int\left(e^{v}-1\right)^{2}$. Thus $\int_{K}\left(e^{v}-1\right)^{2}$. Thus

$$
\left\|\left(e^{v}-1\right)\right\|_{2} \leqq \lim \left\|\left(e^{v_{n}}-1\right)\right\|_{2}, \quad F(v) \leqq \lim F\left(v_{n}\right) .
$$

$F(v)$ is a weakly lower semi-continuous functional on $H_{1}$.

A classical theorem on weakly lower semi-continuous functional is the following

Theorem 4.5. A weakly lower semi-continuous functional on a closed ball in a reflexive Banach space achieves the minimum of the functional on the ball.

Of course the minimum may be achieved on the boundary of the ball. A classical way to exclude this case is to consider the coercivity of the functional.

Theorem 4.6. If a weakly lower semi-continuous functional on a reflexive Banach space is coercive, i.e.,

$$
\lim _{r \rightarrow \infty} \min _{\|v\| \leqq r} F(v)=+\infty .
$$

Then the minimum of the functional is achieved. 
Proposition 4.7. The functional $F(v)$ in (3.6) on the space $H_{1}$ is differentiable with

$$
D_{h} F(v)=\left.\frac{d}{d t}\right|_{t=0} F(v+t h)=\int \nabla v \nabla h+e^{u_{0}+v}\left(e^{u_{0}+v}-1\right) h+g_{0} h .
$$

Proof. The proof is similar to the classical case and is omitted.

Return to the functional

$$
F(v)=\frac{1}{2} \int|\nabla v|^{2}+\left(e^{u_{0}+v}-1\right)^{2}+2 g_{0} v
$$

on the Sobolev space $H_{1}$. We have shown that $F(v)$ is weakly lower semicontinuous and differentiable. We will show that $F(v)$ on $H_{1}$ is a coercive functional and hence $F(v)$ achieves the minimum of $F(v)$ on $H_{1}$ and the minima is a critical point of the functional and a solution to Eq. (3.5) in $H_{1}$. To show $F(v)$ on $H_{1}$ is coercive however needs a detailed analysis on the functional $F(v)$ and which will essentially occupy the rest of the paper.

Consider the functional (4.5), the functional contains three terms, with the nonlinear term having an estimate

$$
\int\left(e^{u_{0}+v}-1\right)^{2}=\int\left(e^{u_{0}}\left(e^{v}-1\right)+\left(e^{u_{0}}-1\right)\right)^{2} \geqq \frac{1}{2} \int e^{2 u_{0}}\left(e^{v}-1\right)^{2}-\int\left(e^{u_{0}}-1\right)^{2},
$$

and we will concentrate on the integral

$$
\int e^{2 u_{0}}\left(e^{v}-1\right)^{2} \text {. }
$$

First note that $e^{2 u_{0}}$ is a potential function which approaches 1 at infinity and vanishes at the zeros of the Higgs field $\phi$ with the same order as $|\phi|^{4}$. This diversity requires different estimates on the integral (4.6) over different areas on the plane.

Consider

$$
R^{2}=\Omega_{0} \cup \Omega_{1}
$$

as a decomposition of the plane with

$$
\Omega_{1}=\left\{x \in R^{2}: e^{u_{0}} \geqq \frac{1}{2}\right\}, \quad \Omega_{0}=\left\{x \in R^{2}: e^{u_{0}} \leqq \frac{1}{2}\right\} .
$$

The function $\left(e^{v}-1\right)^{2}$ is also subject to different behaviors in different cases $v \geqq 0$ and $v \leqq 0$. In the case $v \geqq 0$,

$$
e^{v}-1 \geqq v \geqq 0,
$$

while in the case $v \leqq 0$ by denoting $v_{-}=\max \{0,-v\}$,

$$
1-e^{-v-} \geqq \frac{v_{-}}{1+v_{-}} \geqq 0 .
$$

In both cases the function $\left(e^{v}-1\right)^{2}$ obeys the following:

$$
\left(e^{v}-1\right)^{2} \geqq \frac{|v|^{2}}{(1+|v|)^{2}} .
$$

With the fact that the potential $e^{2 u_{0}}$ is small on the area $\Omega_{0}$ and approaches zero in a certain order in a neighborhood of a zero of the Higgs field, the method we will use to estimate the integral (4.6) is to apply the inverse Hölder inequality. We here introduce the inequality and apply the inequality to estimate the potential integral. The proof of the inverse Hölder is simply by applying the Hölder inequality. 
Theorem 4.8 (Inverse Hölder Inequality). For any measurable functions $f, g$ on an area $\Omega$,

$$
\int_{\Omega}|f g| \geqq\left(\int_{\Omega}|f|^{\alpha}\right)^{1 / \alpha}\left(\int_{\Omega}|g|^{\beta}\right)^{1 / \beta}
$$

with $\alpha, \beta$ are two real numbers, $0<\alpha<1, \beta<0$ and $\frac{1}{\alpha}+\frac{1}{\beta}=1$.

Return to the integral $\int_{\Omega_{0}} e^{2 u_{0}}\left(e^{v}-1\right)^{2}$. On the area $\Omega_{0}$, the potential $e^{2 u_{0}}$ is small and approaching zero in an order at most $4 N$ around a zero of the Higgs field. Thus for any constant $\beta$ with $-\frac{1}{2 N}<\beta<0, \int_{\Omega_{0}} e^{2 u_{0} \beta}$ exists. By applying the inverse Hölder inequality

$$
\begin{aligned}
\int_{\Omega_{0}} e^{2 u_{0}}\left(e^{v}-1\right)^{2} & \geqq \int_{\Omega_{0}} e^{2 u_{0}} \frac{v^{2}}{(1+|v|)^{2}} \geqq\left\{\int_{\Omega_{0}}\left(\frac{|v|}{1+|v|}\right)^{2 \alpha}\right\}^{\frac{1}{\alpha}}\left\{\int_{\Omega_{0}} e^{2 u_{0} \beta}\right\}^{\frac{1}{\beta}} \\
& \geqq C_{6}\left\{\int_{\Omega_{0}}\left(\frac{|v|}{1+|v|}\right)^{2 \alpha}\right\}^{\frac{1}{\alpha}}
\end{aligned}
$$

for some positive constant $C_{6}$ and $\alpha$ with $0<\alpha<1 /(2 N+1)$.

On the other hand, on the area $\Omega_{1}$ with $e^{2 u_{0}} \geqq \frac{1}{2}$,

$$
\int_{\Omega_{0}} e^{2 u_{0}}\left(e^{v}-1\right)^{2} \geqq \frac{1}{2} \int_{\Omega_{1}}\left(\frac{|v|}{1+|v|}\right)^{2} .
$$

Compare the estimates in two different cases: since

$$
\frac{|v|}{1+|v|} \leqq 1, \quad 0<\alpha<1
$$

inequality (4.7) implies

$$
\int_{\Omega_{0}} e^{2 u_{0}}\left(e^{v}-1\right)^{2} \geqq C_{6}\left\{\int_{\Omega_{0}} \frac{v^{2}}{(1+|v|)^{2}}\right\}^{\frac{1}{\alpha}} .
$$

The constant $C_{6}$ depends on the parameter, however, by applying the inequality

$$
x \leqq C_{6} x^{\frac{1}{\alpha}}+C_{7}
$$

for $x \geqq 0$ and for some positive constant $C_{7}$, the integral $\int_{\Omega_{0}} e^{2 u_{0}}\left(e^{v}-1\right)^{2}$ obeys the following estimate:

$$
\int_{\Omega_{0}} e^{2 u_{0}}\left(e^{v}-1\right)^{2} \geqq \int_{\Omega_{0}}\left(\frac{|v|}{1+|v|}\right)^{2}-C_{7} .
$$

Thus the integrals on both areas $\Omega_{0}$ and $\Omega_{1}$ share the following common estimate:

$$
\int e^{2 u_{0}}\left(e^{v}-1\right)^{2} \geqq \frac{1}{2} \int\left(\frac{|v|}{1+|v|}\right)^{2}-C_{7}
$$

Return to the functional

$$
F(v)=\frac{1}{2} \int|\nabla v|^{2}+\left(e^{u_{0}+v}-1\right)^{2}+2 g_{0} v,
$$


by the estimates given above,

$$
F(v) \geqq \frac{1}{2}\|\nabla v\|_{2}^{2}+\frac{1}{8}\left\|\frac{|v|}{1+|v|}\right\|_{2}^{2}-\left\|g_{0}\right\|_{2}\|v\|_{2}-C_{8}
$$

for some positive constant $C_{8}$. To show the coercivity of the functional $F(v)$, we notice that $L^{2}$-norm $\|v\|_{2}$ can be controlled by $\|\nabla v\|_{2}$ and a lower norm by interpolation.

Consider on the space $H_{1}$,

$$
\left(\int v^{2}\right)^{2}=\left(\int \frac{|v|}{1+|v|}|v|(1+|v|)\right)^{2} \leqq \int\left(\frac{|v|}{1+|v|}\right)^{2} \int v^{2}(1+|v|)^{2} \leqq 2 \int\left(\frac{|v|}{1+|v|}\right)^{2} \int\left(v^{2}+v^{4}\right)
$$

and the integral $\int v^{4}$ can be estimated by the following lemma.

Lemma 4.9. On $H_{1}\left(R^{2}\right)$ the following inequality holds:

$$
\int v^{4} \leqq \int v^{2} \int|\nabla v|^{2}
$$

Proof. On $R^{2}$, any $L_{1}^{1}$ function $w$ satisfies

$$
\|w\|_{2} \leqq \frac{1}{2} \int|\nabla w|
$$

(see cf. Chap. 2 of [1]). Let $w=v^{2}$ then the substitution and Hölder inequality imply the lemma.

Hence

$$
\begin{aligned}
\left(\int v^{2}\right)^{2} & \leqq 2 \int\left(\frac{|v|}{1+|v|}\right)^{2}\left(\int v^{2}\right)\left(1+\int|\nabla v|^{2}\right) \\
\int v^{2} & \leqq 2\left\|\frac{|v|}{1+|v|}\right\|_{2}^{2}\left(1+\int|\nabla v|^{2}\right) \\
\|v\|_{2} & \leqq \sqrt{2}\left\|\frac{|v|}{1+|v|}\right\|_{2}\left(1+\|\nabla v\|_{2}\right) \\
\|v\|_{2} & \leqq\left\|\frac{|v|}{1+|v|}\right\|_{2}^{2}+2\|\nabla v\|_{2}^{2}+2 .
\end{aligned}
$$

Using Lemma 4.1, the functional $F(v)$ on $H_{1}$ enjoys the following estimate:

$$
\begin{aligned}
F(v) \geqq & \frac{1}{2}\|\nabla v\|_{2}^{2}+\frac{1}{8}\left\|\frac{|v|}{1+|v|}\right\|_{2}^{2}-\frac{C_{0}}{\sqrt{\mu}}\|v\|_{2}-C_{8} \geqq \frac{1}{2}\|\nabla v\|_{2}^{2}+\frac{1}{8} \| \frac{|v|}{1+|v| \|_{2}^{2}} \\
& -\frac{C_{9}}{\sqrt{\mu}}\left(\left\|\frac{|v|}{1+|v|}\right\|_{2}^{2}+\|\nabla v\|_{2}^{2}\right)-C_{10}
\end{aligned}
$$

for some positive constants $C_{9}$ and $C_{10}$. Therefore when the parameter $\mu$ is chosen to be large, there are positive constants $C_{11}, C_{12}$ such that

$$
F(v) \geqq C_{11}\|\nabla v\|_{2}^{2}+C_{12}\left\|\frac{|v|}{1+|v|}\right\|_{2}^{2}-C_{10} .
$$

Applying inequality (4.8),

$$
F(v) \geqq C_{13}\|\nabla v\|_{2}^{2}+C_{14}\|v\|_{2}-C_{15} \geqq C_{16}\|v\|-C_{17}
$$

for positive constants $C_{13}$ to $C_{17}$. 
As a consequence of the estimate (4.8), the infimum of $F(v)$ is finite and $F(v)$ is coercive on the Banach space $H_{1}$.

Theorem 4.10. The functional $F(v)$ achieves the minimum on the Banach space $H_{1}$.

Proof. This is an immediate corollary of Theorem 4.5.

The differentiability of the functional $F(v)$ on $H_{1}$ assures that the minima of $F(v)$ satisfies the Euler-Lagrange equation (3.5) and the standard elliptic regularity theory can be used to show that the solution $v$ in $H_{1}$ is smooth. Back to Eq. (2.5), combining Theorem 2.1, $\phi=e^{\frac{1}{2}(u+i \theta)}, \alpha=i \partial_{z} \ln \bar{\phi}$ is a smooth Chern-Simons vortex solution with zeros exactly at $z_{1}, \ldots, z_{n}$. The Theorem in the Introduction is proved.

The last word we will say is the fact that it is easy to see that the solution to the Euler-Lagrange equation (3.5) obeys an a priori estimate $v+u_{0}<0$ by the maximum principle which is equivalent to Theorem 2.2.

\section{References}

1. Aubin, T.: Nonlinear analysis on manifolds. Monge-Ampere equations. Berlin, Heidelberg, New York: Springer 1982

2. Bradlow, S.: Vortices on Kähler manifolds. Thesis, University of Chicago, 1988

3. Deser, S., Jackiw, R., Templeton, S.: Topological massive gauge theories. Ann. Phys. 140, 372411 (1982)

4. Chen, Y.Y.: Vortices for the Ginzburg-Landau equations - the nonsymmetric case in bounded domain. AMS Contemporary Mathematics, Number 108, 1990

5. Evans, L.C.: Weak convergence method for nonlinear partial differential equations. AMS Regional Conference Series in Mathematics, Number 74

6. Fröhlich, J., Marchetti, P.A.: Quantum field theories of vortices and anyons. Commun. Math. Phys. 121, 177-223 (1989)

7. Gilbarg, D., Trudinger, N.: Elliptic partial differential equations of second order. Second Ed. Berlin, Heidelberg, New York: Springer 1983

8. Ginzburg, V.L., Landau, L.D.: On the theory of superconductivity. Zh. Eksper. Theoret. Fiz. 20, 1064-1073 (1950)

9. Hagen, C.R.: A new gauge theory without an elementary Photon. Ann. Phys. 157, 342-359 (1984)

10. Jackiw, R., Weinberg, E.J.: Self-dual Chern-Simons vortices. Preprint submitted to Phys. Lett., Feb. 1990

11. Jackiw, R., Pi, S.-Y.: Gauged nonlinear Schrödinger equation on the plane. Preprint submitted to Phys. Lett., June 1990

12. Jackiw, R., Pi, S.-Y.: Classical and quantal nonrelativistic Chern-Simons theory. Preprint submitted to Phys. Rev. D 15, June 1990

13. Morry, C.B., Jr.: Multiple integrals in the calculus of variations. Berlin, Heidelberg, New York: Springer 1966

14. Jaffe, A., Taubes, C.: Vortices and monopoles. Boston: Birkhäuser 1980

15. Taubes, C.: Arbitrary $n$-vortex solutions to the first order Ginzburg-Landau equations. Commun. Math. Phys. 72, 277-292 (1980)

16. Schiff, J.: Integrability of Chern-Simons-Higgs and abelian Higgs equations in a background metric. Preprint submitted to Phys. Lett., March 1990

17. Smoller, J., Yau, S.-T.: To appear in the collected volumes on UCLA Summer School in Geometry, 1990

18. Hong, J., Kim, Y., Pac, P.Y.: Phys. Rev. Lett. 64, 2230 (1990) 
